\title{
Simultaneous Fine and Coarse Diffeomorphic Registration: Application to Atrophy Measurement in Alzheimer's Disease
}

\author{
Laurent Risser ${ }^{1,2}$, François-Xavier Vialard ${ }^{1}$, Robin Wolz $^{2}$, \\ Darryl D. Holm ${ }^{1}$, and Daniel Rueckert ${ }^{2}$ \\ 1 Institute for Mathematical Science, Imperial College London, \\ 53 Prince's Gate, SW7 2PG, London, $\mathrm{UK}^{\star}$ \\ 2 Visual Information Processing, Imperial College London, Huxley Building, \\ Department of Computing, SW7 2BZ, London, UK
}

\begin{abstract}
In this paper, we present a fine and coarse approach for the multiscale registration of 3D medical images using Large Deformation Diffeomorphic Metric Mapping (LDDMM). This approach has particularly interesting properties since it estimates large, smooth and invertible optimal deformations having a rich descriptive power for the quantification of temporal changes in the images. First, we show the importance of the smoothing kernel and its influence on the final solution. We then propose a new strategy for the spatial regularization of the deformations, which uses simultaneously fine and coarse smoothing kernels. We have evaluated the approach on both 2D synthetic images as well as on $3 \mathrm{D}$ MR longitudinal images out of the Alzheimer's Disease Neuroimaging Initiative (ADNI) study. Results highlight the regularizing properties of our approach for the registration of complex shapes. More importantly, the results also demonstrate its ability to measure shape variations at several scales simultaneously while keeping the desirable properties of LDDMM. This opens new perspectives for clinical applications.
\end{abstract}

\section{Introduction}

Recent years have seen the development of new non-rigid registration techniques allowing large diffeomorphic deformations. Such deformations are by definition smooth and invertible, properties that are highly desirable in image registration. In particular, the framework of Large Deformation Diffeomorphic Metric Mapping (LDDMM) 4,10] encodes the optimal flows of deformation in timedependent velocity fields, that are geodesic. The optimal flow between two registered images is then the shortest path between the images according to a metric regularizing the deformation. The LDDMM approach has therefore convenient properties for the statistical comparison of images and the creation of atlases.

\footnotetext{
* We thank the Imperial College Strategic Initiative Fund for partial support. DDH was also partially supported by the Royal Society of London Wolfson Research Merit Award. We are finally thankful to Martins Bruveris for constructive discussions.
} 
A practical implementation of the this framework for image registration has been proposed in [4 and successfully applied to inter-subject local shape comparison and atlas creation. However, although it was designed to allow large deformations, its practical use in real medical images is often limited to relatively small deformations. Alternatives approaches have been proposed in the literature: For instance, a symmetric interpretation of [4] using cross correlation to measure the similarity between source and target images was proposed in 3 . Other approaches, allowing multimodal registration for atlas creation [9] or using Navier-Stokes equation 5] were also proposed. Importantly, a class of diffeomorphic registration techniques, using stationary velocity fields, have emerged recently. Such parameterizations are efficient in terms of memory requirements and computational time while providing registrations similar to those obtained using the LDDMM time-dependent velocity fields [1,2,8,13. However, although these alternatives also estimate diffeomorphic deformations, none of them has been explicitly designed to estimate geodesic transformations.

In an attempt to improve the practical usability of LDDMM for 3D medical imaging, we address in this paper a fundamental aspect of the LDDMM framework: the choice of the metric. The metric is indeed directly related to the smoothing kernel of the deformations and therefore controls their spatial regularization. In practice, small kernels will favor deformations that match local details and large kernels deformations that match global structures. Since 3D medical images may contain complex anatomical structures, with features varying at different scales, multiscale registration techniques are of high interest. However, the classical coarse to fine strategies based on Gaussian kernels are theoretically (and practically) not appropriate in the context of LDDMM since the registration at the final scale only reflects the shape variations at the finest scale. The key contribution here is then the development of a theoretically well-justified simultaneous fine and coarse registration strategy in the LDDMM framework. The idea underlying this paper is related to the strategy proposed in [7] which consists in registering images at several scales simultaneously.

In Sec. 2, we present our multiscale extension of 4 . Importantly, we show that this extension is particularly suitable to describe the deformations of shapes whose features exist across several scales. We then discuss in Sec. 3, its behaviour for registrations requiring large deformations and its ability to detect shape variations at several scales simultaneously. We finally present the usability of our approach on medical images by comparing 3D MR longitudinal images out of the Alzheimer's Disease Neuroimaging Initiative (ADNI) study.

\section{Registration Using Multi-kernel LDDMM}

\subsection{Registration Using LDDMM}

We first give a brief summary of the classical LDDMM registration algorithm [4]. Let $I_{S}$ be a source image, defined on a spatial domain $\Omega$, and registered on a target image $I_{T}$ through the time-dependent diffeomorphic transformation $\phi_{t}$ of $\Omega, t \in[0,1]$. This transformation is defined by a time dependent velocity field $v_{t}$, 
$t \in[0,1]$ as follows: $\partial_{t} \phi_{t}=v_{t}\left(\phi_{t}\right)$, where $\phi_{0}=I d$ and $t \in[0,1]$. For notational convenience we introduce $\phi_{t, s} \doteq \phi_{s} \circ \phi_{t}^{-1}$. The registration problem then consists in finding the velocity fields $v_{t}$ that minimizes the sum of a similarity and a deformation energy:

$$
E(v)=\int_{0}^{1} \frac{1}{2}\left\|v_{t}\right\|_{V}^{2} \mathrm{~d} t+\frac{1}{2}\left\|I_{S} \circ \phi_{1}^{-1}-I_{T}\right\|_{L^{2}}^{2} .
$$

The second term measures the similarity between the deformed source and target images, here, the sum of squared differences of the intensities in both images. To define the energy of the deformation, the time dependent velocity field $v$ is assumed to lie in $L^{2}([0,1], V)$, where the Hilbert space $V$ is expressed by a smooth matrix valued kernel $k(.,$.$) describing the velocity fields that can be$ used for the registration. Even though there is a wide family of available kernels associated with $V$, most approaches use Gaussian kernels:

$$
K(x)=(2 \pi)^{-d / 2}|\Sigma|^{-1 / 2} \exp \left(-\frac{1}{2} x^{T} \Sigma^{-1} x\right),
$$

where $\Sigma$ is the covariance matrix of the Gaussian kernel. In our work, we assume isotropic covariances, i.e. $\Sigma=\sigma I d_{\mathbb{R}^{d}}$, where the key parameter $\sigma$ controls the level of the spatial correlation of the deformations. The minimization algorithm is described hereafter. We denote $J_{t}^{S}=I_{S} \circ \phi_{t, 0}, J_{t}^{T}=I_{T} \circ \phi_{1, t}$ and $\left|D \phi_{t, 1}\right|$ the Jacobian of $\phi_{t, 1}$ at time $t$. The minimization of the variational problem of eq. 1 is performed by using a steepest gradient descent approach. This involves the iterative use of the gradient of $E$ in $L^{2}([0,1], V), \forall t$ :

$$
\nabla_{v} E_{t}=v_{t}-K \star\left(\left|D \phi_{t, 1}^{v}\right| \nabla J_{t}^{S}\left(J_{t}^{S}-J_{t}^{T}\right)\right),
$$

where $\star$ denotes the convolution operator. The velocity field is then updated by computing $v^{k+1}=v^{k}-\epsilon \nabla_{v_{t_{j}}^{k}} E$, where $\epsilon$ controls the step size during the gradient descent. After convergence towards the minimum energy solution, the resulting time dependent diffeomorphism is a geodesic path in the group of diffeomorphisms for which the associated velocity field satisfies the Euler-Lagrange equation.

\subsection{Multi-kernel LDDMM}

As discussed in introduction, the comparison of shapes can only be performed at a single scale when using [4 with classical Gaussian kernels. To perform the registration at several scales simultaneously, we simply define the kernel $K$ as the weighted sum of $N$ Gaussian kernels $K_{n}$ of different sizes as follows:

$$
K(x)=\sum_{n=1}^{N} K_{n}(x)=\sum_{n=1}^{N} a_{n}(2 \pi)^{-3 / 2}\left|\Sigma_{n}\right|^{-1 / 2} \exp \left(-\frac{1}{2} x^{T} \Sigma_{n}^{-1} x\right),
$$

where $\Sigma_{n}$ and $a_{n}$ are respectively the covariance matrix and the weight of the $n^{\text {th }}$ Gaussian function. As in eq. 2 each $\Sigma_{n}$ is only defined by a characteristic 


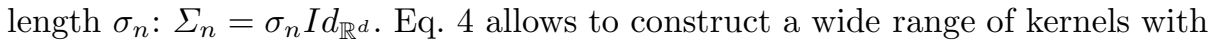
several scales of interest while preserving the promising statistical properties of the LDDMM. The optimization is then performed simultaneously at several scales so that complex shapes can be compared in this framework. Note that the choice of the weights $\left(a_{n}\right)_{n \in[1, N]}$ (discussed in more detail in sec. 2.4) is a key issue here, since it controls the influence of the deformations at different scales.

\subsection{Separating the Contribution of Each Kernel}

Interestingly, our multi-kernel extension of [4] can be re-written to distinguish the contribution of each kernel $K_{n}$ in the total deformation. A velocity field $v_{n}$ is associated with each kernel $K_{n}$. The energy gradient described in eq. 3 becomes:

$$
\nabla_{v_{n}} E_{t}=v_{n, t}-K_{n} \star\left(\left|D \phi_{t, 1}^{v}\right| \nabla J_{t}^{S}\left(J_{t}^{S}-J_{t}^{T}\right)\right), \quad \forall n,
$$

and the velocity field is updated by using:

$$
v_{n}^{k+1}=v_{n}^{k}-\epsilon \nabla_{v_{n, t_{j}}^{k}} E, \quad \forall n .
$$

This formulation of the gradient descent is equivalent to the original one and provides the same deformations. Using the equations 5 and 6, the sum of the fields $v_{n}$ is indeed equal to the total velocity field: $v=\sum_{n=1}^{N} v_{n}$. The deformation of the source image can also be computed using $\partial_{t} \phi_{t}=\sum_{n=1}^{N} v_{n, t}\left(\phi_{t}\right)$ and $\phi_{0}=$ $I d$. Observing the contribution of a kernel $K_{n}$ at a point $x$ of the deformed image is then naturally done by observing the amplitude of the deformation (AOD) generated by $v_{n, t}$ along $\phi_{t}(x), t \in[0,1]: A O D_{n}(x)=\int_{0}^{1}\left|v_{n, t}\left(\phi_{t} \circ x\right)\right| \mathrm{d} t$. Obviously, using $v_{t}$ instead of $v_{n, t}$ in this equation gives the length of the total deformation from $x$. As seen in section 3.2, the AOD is an interesting descriptor of the local deformations for shapes whose features exist across several scales.

\subsection{Weight of the Kernels}

Our multiscale strategy depends on a set of parameters $a_{n}, n \in[1, N]$, each of them controlling the weight of the deformations at scale $n$. These weights reflect indirectly the amplitude of the deformations expected between the different scales. We introduce the apparent weights $a_{n}^{\prime}, n \in[1, N]$ that give to the user an intuitive control of the deformation at each scale. Importantly, similar values of $a_{n}^{\prime}$ induce deformations having similar amplitudes at each scale $n$. The weights are then computed using $a_{n}=a_{n}^{\prime} / g_{n}\left(K_{n}, I_{S}, I_{T}\right)$ where $g_{n}$ is the typical amplitude of the updates in eq. 6 between $I_{S}$ and $I_{T}$, if $K_{n}$ has a weight equal to 1 . For each $K_{n}$, the value of $g_{n}$ can be estimated by observing the maximum update of the velocity field $v$ in a pre-iteration of the registration of $I_{S}$ on $I_{T}$ using only the kernel $K_{n}$ with $a_{n}=1$. This simple technique is shown to work efficiently both on 2D synthetic images and 3D medical images. More importantly, for images of the same type (e.g. MR brain images with a fixed acquisition protocol) and the same kernels $K_{n}$, the values of $g_{n}$ were observed stable. The method can then be used for an atlas creation or multiple comparisons by systematically using the same kernels with same weights. 


\section{Evaluation}

\subsection{Application to 2D Synthetic Images}

As discussed above, the kernel controls the spatial behavior of the deformations. For an isotropic Gaussian kernel with standard deviation $\sigma$, the parameter $\sigma$ is the characteristic length defining the scale at which the registration is performed. To illustrate the behavior of the deformation as a function of the kernel, we show in Fig. 1 an example of deformation in which a square $\left(I_{S}\right)$ is registered to a rectangle containing an indentation $\left(I_{T}\right)$ using a small kernel $(\sigma=1.5$ pixels), a large kernel ( $\sigma=5$ pixels) and the sum of kernels with the same apparent weights. Note that the deformation grid obtained using the small kernel seems to highlight a non-invertible deformation. Refining this grid shows that the deformation is actually invertible but with very high Jacobians.

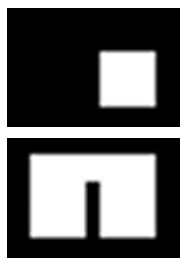

(a)

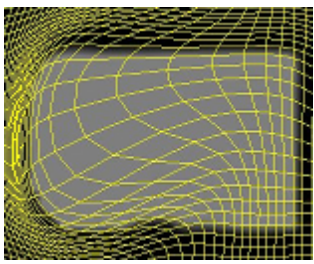

(b)

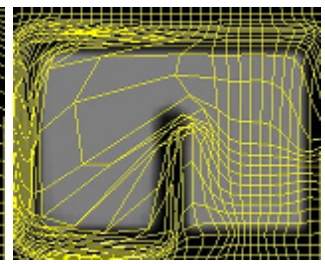

(c)

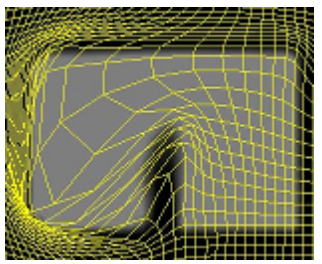

(d)

Fig. 1. Large diffeomorphic deformation of a 2D binary image. (a) Source image $I_{S}$ and target image $I_{T}$ and deformations using: (b) a large kernel, (c) a small kernel and (d) the sum of these kernels.

As expected, at a large scale the square is registered on the rectangle with a small perturbation while at a small scale the registration fully resolves the shape differences. Using the sum of these kernels, the deformation appears relatively similar to the one obtained using only the small kernel, even though the matching quality is slightly lower. However, as shown in the deformation grids of Fig. 1 the multi-kernel approach generates deformations that are clearly smoother and more intuitive than those obtained using the small kernel for similar final deformation. This is due to the simultaneous consideration of two scales all along the flow of deformation. Hence, in order to match objects with several scales of interest, our multi-kernel technique appears efficient since the registration of small and large scales is treated simultaneously with appropriate kernels. More discussions are given in [1].

\subsection{Application to 3D Brain MR Images}

To assess the ability of the proposed method in dealing with medical images, we applied it to the discrimination of MR brain images of patients with Alzheimer's disease $(\mathrm{AD})$ and healthy age-matched controls. For each subject group, we 
downloaded 30 pairs of images taken at baseline and after 24 months follow-up from the Alzheimer's Disease Neuroimaging Initiative (ADNI) study [12. All T1weighted 1.5T MR-images were pre-processed using the standard ADNI pipeline. We aligned all images with the MNI152 brain template using affine registration before extracting a region of interest of $128^{3}$ voxels around the hippocampus. By using this strategy instead of more accurate intra-subject pre-registrations, the baseline and follow-up images present variations due to the atrophy of the tissues and due to the inaccurate pre-registration. Therefore, comparing them at large and small scales simultaneously and quantifying the differences at small scale only is pertinent. For each subject, we then registered the baseline and follow-up MR images using several strategies: (LK) Using a large kernel of characteristic size $\sigma_{1}=25 \mathrm{~mm}$ which is obviously higher than the deformations we want to measure, (SK) using a small kernel of characteristic size $\sigma_{2}=1.5 \mathrm{~mm}$ closer to the apparent transformations, (SGK) using the sum of these Gaussian kernels with apparent weights $a_{2}^{\prime}=2 a_{1}^{\prime}$ and means of $g_{1}$ and $g_{2}$ in each group, and finally (LSK) using the small kernel $\sigma_{1}$ to register the deformed baseline images resulting from the deformation (LK), with the follow-up image. This last strategy corresponds to a coarse to fine approach.
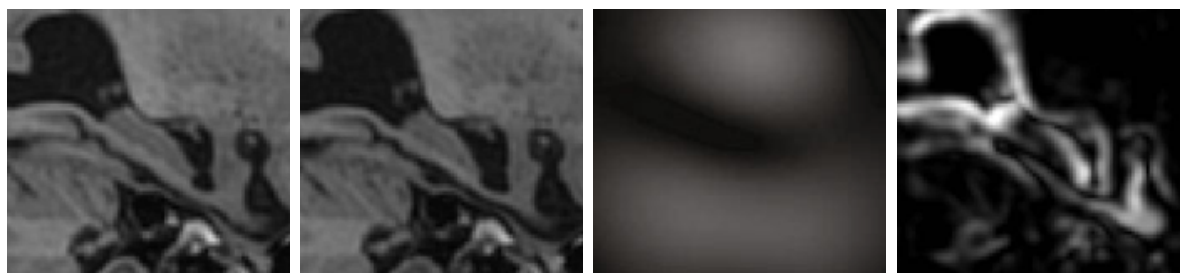

Fig. 2. (Left) Baseline and follow-up MR images of the hippocampus out of an AD subject. (Right) AOD using the sum of kernel. The contributions $\mathrm{SGK}_{1}$ and $\mathrm{SGK}_{2}$ of the large and small kernels is separated here.

We compared the temporal changes in hippocampus using several descriptors by automatically segmenting the hippocampus in all baseline images and transforming these volumes using the flow of deformations resulting from the registration of the grey level images. We measured, for each subject, the hippocampal atrophy using: $A=\left(V_{s}-V_{t}\right) / V_{s}$, where $V_{s}$ and $V_{t}$ are the hippocampal volumes in the segmented baseline image and after deformation to the second time point respectively. We also measured the amplitude of the deformations (AOD) from each point $x$ of the hippocampal surface and separated the contributions of the large $\left(S G K_{1}\right)$ and the small $\left(S G K_{2}\right)$ kernels for (SGK) using the technique described in subsection 2.3. Here the AOD have been non-dimensionalized by the cubic root of $V_{s}$ to remove the bias due to inter-subject variations of the initial hippocampal volume. For comparison purpose, we also computed from the same points of the surface the determinant of the Jacobian $(\operatorname{det}(\mathrm{J})$ ) of the final deformations $\phi_{1}$. Group-dependent average values and P-values of 
non-parametric Mann-Whitney tests between the groups, which measure the discriminative power of each descriptor, are presented in table 1 .

The global measure of the atrophy is similar to the measures reported in the literature 12 for (LSK) and (SGK) and turns out to be the most discriminant descriptor. The local measures of displacement AOD and $\operatorname{det}(\mathrm{J})$, averaged on the surfaces, appear to have a similar discriminant power, with slightly better results for AOD. In AOD: (LK) The differences between the groups reflect the variations at large scale but the influence of the small scales is not completely negligible. (SK) The discrimination is surprisingly good but biased by the deformations at large scale. (LSK) The discrimination is good. (SGK) The discriminative power is in between (LK) and (LSK) if the deformations $\left(\mathrm{SGK}_{1}\right)$ and $\left(\mathrm{SGK}_{2}\right)$ are not distinguished. However, when separated, the large structures are registered with few influence of the small ones and vice-versa so the discrimination is better using $\left(\mathrm{SGK}_{2}\right)$ than $(\mathrm{LSK})$. Note that by using $a_{2}^{\prime}=a_{1}^{\prime}$ the results are similar with a slightly smaller discriminative power of $\mathrm{SGK}_{2}$. Similarly, in LSK the image is resampled after the registration at large scale. The results would be improved by integrating the first deformation in $\phi_{0}$ instead.

Table 1. Average values of atrophy, AOD and determinant of the Jacobian in the hippocampal, observed in the $\mathrm{AD}$ and healthy $(\mathrm{H})$ groups. $\mathrm{P}$-values of non-parametric Mann-Whitney tests measure the discriminative power of each descriptor. The lower the P-value, the clearer the distinction.

\begin{tabular}{|c|c|c|c|c|c|c|c|c|c|}
\hline & \multicolumn{3}{|c|}{ \% Atrophy } & \multicolumn{3}{c|}{ Average AOD } & \multicolumn{3}{c|}{ Average det(J) } \\
\cline { 2 - 11 } & mean AD & mean H & P-val. & mean AD & mean H & P-val. & mean AD & mean H H-val. & P-val \\
\hline \hline LK & 1.9 & 1.4 & 0.68 & 0.67 & 0.56 & 0.054 & 0.99 & 0.99 & 0.95 \\
\hline SK & 8.2 & 1.4 & $1.3 \mathrm{e}-6$ & 0.57 & 0.30 & $2.2 \mathrm{e}-5$ & 0.96 & 0.99 & $2.1 \mathrm{e}-4$ \\
\hline LSK & 9.6 & 2.8 & $1.9 \mathrm{e}-6$ & 0.51 & 0.29 & $2.8 \mathrm{e}-4$ & 0.96 & 0.98 & 0.06 \\
\hline SGK & 8.1 & 2.0 & $3.6 \mathrm{e}-7$ & 0.72 & 0.52 & $1.7 \mathrm{e}-3$ & 0.96 & 0.99 & $6.9 \mathrm{e}-5$ \\
\hline \hline SGK $_{1}$ & - & - & - & 0.47 & 0.46 & 0.49 & - & - & - \\
\hline SGK $_{2}$ & - & - & - & 0.41 & 0.20 & $2.4 \mathrm{e}-6$ & - & - & - \\
\hline
\end{tabular}

\section{Conclusion}

We have presented an approach of fine and coarse registration in the context of LDDMM for 3D medical images. This approach is of particular interest since the images often contain anatomical structures that exhibit variations at several scales of interest that cannot be properly measured using a simple or a coarse to fine LDDMM registration. The approach we developed makes use of the sum of several Gaussians to define the kernel underlying the deformations. Our tests have shown that this method estimates natural-looking deformations when registering images presenting variations at two different scales while keeping diffeomorphic properties. More importantly, it appears that our fine and coarse approach may have better abilities than standard coarse to fine approaches to 
discriminate two groups of images by measuring the amplitudes of the deformations generated at a scale of interest. Note, that these scale-dependent amplitudes of deformations are local, so localized measures of shape evolution can be performed. Future work will consist in the validation of a multi-resolution approach and the assessment of the geodesic properties of the estimated deformations. A deeper characterization of the apparent weights is also an important perspective of this work. More experiments and applications of our technique will also be carried out on MR cerebral images as well as on CT cardiac images.

\section{References}

1. Arsigny, V., Commowick, O., Pennec, X., Ayache, N.: A log-Euclidean framework for statistics on diffeomorphisms. In: Larsen, R., Nielsen, M., Sporring, J. (eds.) MICCAI 2006. LNCS, vol. 4190, pp. 924-931. Springer, Heidelberg (2006)

2. Ashburner, J.: A fast diffeomorphic image registration algorithm. NeuroImage 38, 95-113 (2007)

3. Avants, B.B., Epstein, C.L., Grossman, M., Gee, J.C.: Symmetric diffeomorphic image registration with cross-correlation: Evaluating automated labeling of elderly and neurodegenerative brain. Medical Image Analysis 12, 26-41 (2008)

4. Beg, F.M., Miller, M.I., Trouvé, A., Younes, L.: Computing large deformation metric mappings via geodesic flows of diffeomorphisms. International Journal of Computer Vision 61(2), 139-157 (2005)

5. Crum, W., Tanner, C., Hawkes, D.: Anisotropic multi-scale fluid registration: evaluation in magnetic resonance breast imaging. Physics in Medicine and Biology 50(21), 5153-5174 (2005)

6. Dupuis, P., Grenander, U., Miller, M.I.: Variational problems on flows of diffeomorphisms for image matching. Q. Appl. Math. LVI(3), 587-600 (1998)

7. Haber, E., Modersitzki, J.: Cofir: coarse and fine image registration. In: SIAM Real-Time PDE-Constrained Optimization, pp. 37-49 (2007)

8. Hernandez, M., Bossa, M.N.,Olmos, S.: Registration of anatomical images using paths of diffeomorphisms parameterized with stationary vector field flows. Int. J. Comput. Vision 85(3), 291-306 (2009)

9. Lorenzen, P., Prastawa, M., Davis, B., Gerig, G., Bullitt, E., Joshi, S.: Multi-modal image set registration and atlas formation. Med. Image Anal. 10(3), 440-451 (2006)

10. Miller, M., Younes, L.: Group actions, homeomorphisms, and matching: A general framework. International Journal of Computer Vision 41(1-2), 61-84 (2001)

11. Risser, L., Vialard, F., Murgasova, M., Holm, D., Rueckert, D.: Large diffeomorphic registration using fine and coarse strategies. application to the brain growth characterization. In: International Workshop on Biomedical Image Registration -WBIR, pp. 186-197 (2010)

12. Schuff, N., Woerner, N., Boreta, L., Kornfield, T., Shaw, L.M., Trojanowski, J.Q., Thompson, P.M., Jack, J.C.R., Weiner, M.W.: Disease Neuroimaging Initiative: MRI of hippocampal volume loss in early Alzheimer's disease in relation to ApoE genotype and biomarkers. Brain 132(4), 1067-1077 (2009)

13. Vercauteren, T., Pennec, X., Perchant, A., Ayache, N.: Diffeomorphic demons: Efficient non-parametric image registration. NeuroImage 45(1), S61-S72 (2009) 